\title{
Efficiency Potential of Solid-
} State Pulse Modulators using SiC

\section{Devices}

\section{Conference Paper}

\section{Author(s):}

Stathis, Spyridon; Jaritz, Michael; Blume, Sebastian; Biela, Jürgen (D)

Publication date:

2020

Permanent link:

https://doi.org/10.3929/ethz-b-000495892

Rights / license:

In Copyright - Non-Commercial Use Permitted

Originally published in:

https://doi.org/10.23919/epe20ecceeurope43536.2020.9215760 


\title{
Efficiency Potential of Solid-State Pulse Modulators using SiC Devices
}

\author{
Spyridon Stathis, Michael Jaritz, Sebastian Blume and Jürgen Biela \\ Laboratory for High Power Electronic Systems, ETH Zürich, Switzerland \\ Email: stathis@hpe.ee.ethz.ch
}

\section{Keywords}

"Pulse Transformer", "Resonant Converter", "Silicon Carbide (SiC)", "Power Factor Correction (PFC) Converter", "Solid-State Pulse Modulator", "Efficiency Calculation"

\begin{abstract}
In this paper, the efficiency of the CLIC solid-state pulse modulator developed for the CERN research center and the long-pulse modulator system built for the ESS facility is investigated in detail. The analysis shows the current efficiency status of the modulators and how their performance could be improved by using SiC devices.
\end{abstract}

\section{Introduction}

Pulse modulator systems are key elements in many application areas such as cancer treatment, particle accelerators and radar systems. The efficiency of these systems becomes more and more important as an increasing number of accelerator facilities try to reduce the greenhouse gas emissions [1-3]. Pulse modulators, which are based on solidstate switches, have been proven to be able to efficiently generate pulses at high power levels [4],[5]. Typically, IGBT modules are used as solid-state switches as they offer high blocking voltages and current ratings, and these have demonstrated a reliable operation in pulsed power applications [6].

The solid-state pulse modulators, typically, consist of several power conversion stages between the grid and the load and each stage contributes to the overall system losses. However, so far, only a few publications have investigated the efficiency of the complete modulator system [1],[3]. Also, the impact of using wide bandgap devices, such as $\mathrm{SiC}$, on the system efficiency has been rarely examined [7]. Hence, in this paper, a comprehensive investigation of the efficiency of two solid-state pulse modulators as well as the efficiency potential using SiC devices are presented.

In the first step, the efficiency of each conversion stage of the CLIC solid-state pulse modulator presented in [8], which is based on Si semiconductors, is calculated and the total efficiency is determined. Afterwards, the efficiency

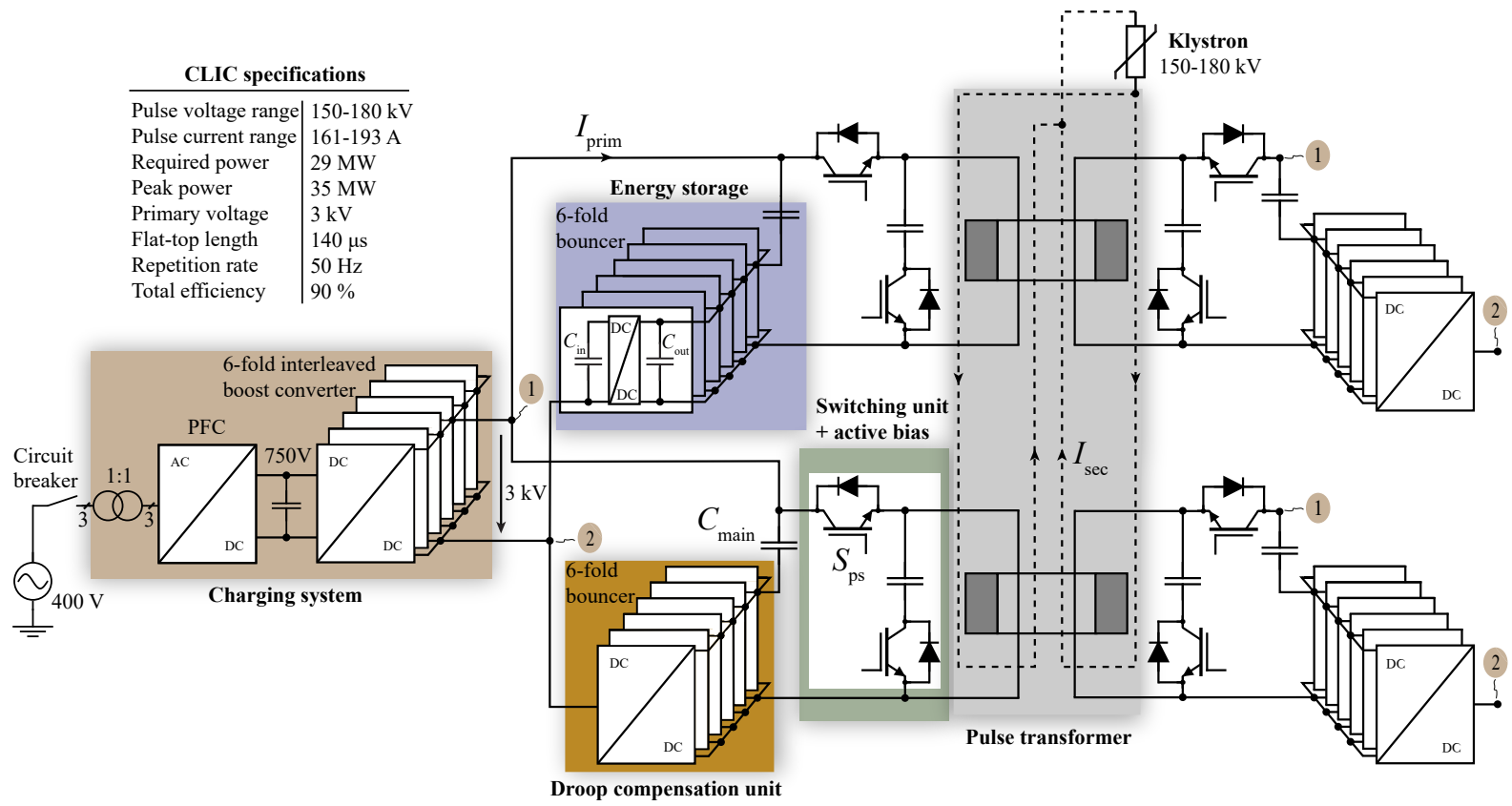

Fig. 1: CLIC pulse modulator schematic overview consisting of: Charging system, droop compensation units along with the main capacitors (energy storage), switching + active bias units, pulse transformer, and klystron load. 
improvements by using $\mathrm{SiC}$ devices, along with other suggested improvements, is evaluated. Similarly, the efficiency of each sub-system along with the investigation of the impact of using SiC devices is presented for the long-pulse modulator developed for the ESS linear accelerator [9]. The SiC-based systems are not re-optimized in terms of efficiency, but a 1:1 replacement of the power switches is assumed to evaluate the impact of the SiC devices. Further improvements are possible in case an optimization is performed for the SiC-based systems.

In the following section 2, the structure of the CLIC system is explained and the main specifications of the pulse modulator are given. In section 3, the efficiency and the distribution of the losses in each stage are derived for the existing Si-based and for the improved SiC-based modulator system. In section 4, the total efficiency for the Si-based and the SiC-based CLIC modulator is extracted, the contribution of each stage to the total efficiency is presented and in the end the results are discussed. In section 5, the topology and the main specifications of the ESS modulator are shown while in section 6, the loss share of each stage of the current Si-based and the improved $\mathrm{SiC}$-based system is provided. In section 7, the total efficiency is given for the Si-based and the SiC-based ESS modulator and the results are evaluated. Finally, section 8 summarizes the main outcomes of the present work.

\section{CLIC Modulator Topology}

A schematic overview of the CLIC modulator topology is shown in Fig. 1. The main specifications of the system are also listed in the figure. At the input, a charging system is connected to the 3-phase $400 \mathrm{~V}$ AC grid which charges the four main capacitors $C_{\text {main }}$ up to $3 \mathrm{kV}$. The charging system consists of a 1:1 isolation transformer, a power factor correction (PFC) rectifier and a 6-fold interleaved boost converter. Four droop compensation units are connected in series to the four main capacitors in order to compensate the droop during the pulse interval. Each droop compensation unit consists of six interleaved buck-boost modules which are referred as bouncer modules. The four switching units $S_{\mathrm{ps}}$, which also include an active bias circuit to pre-magnetize the core of the pulse transformer, connect the primary winding of the pulse transformer to the main capacitor. A non-linear load, the klystron, is attached to the secondary side of the split two-core pulse transformer [2].

\section{Efficiency of CLIC Stages}

In the following, the efficiency of each stage is derived for the Si-based and the improved SiC-based modulator. It is noted that the efficiency calculation is based on the maximum junction temperatures that the semiconductors can withstand. The analysis begins with the efficiency calculation of the charging system.

\section{Charging system}

The efficiency derivation for the charging system is split into its three sub-systems, namely the isolation transformer, the PFC rectifier and the boost converter.

- Isolation transformer: The isolation transformer is a 3-phase $250 \mathrm{kVA}$ unit. The loss distribution for the transformer of the existing modulator can be seen in table I. These values are taken from the data sheet. The core material of the transformer is silicon steel while the windings are made of aluminium. However, if copper windings are used, then the conductivity becomes higher and the winding losses can be reduced by approximately 35\%. In addition, if an amorphous core material is used, such as steel with $25 \mu \mathrm{m}$ ribbon thickness, the core losses can be reduced by $60 \%$ [10].

- PFC rectifier: For the PFC rectifier, two ACOPOSmulti 8BVP1650 active rectifiers in parallel connection are used [11]. Each active rectifier is rated for $120 \mathrm{~kW} / 750 \mathrm{~V} \mathrm{DC}$. The rectifier unit is based on $1.2 \mathrm{kV} \mathrm{Si}$ IGBTs and it has an efficiency of $97.5 \%$ according to the data sheet. However, even a $99 \%$ efficiency is feasible to be achieved for the PFC unit in case SiC technology is adopted [12],[13].

- 6-fold interleaved boost converter: For charging the main capacitors, a six-fold interleaved boost converter, which operates under boundary conduction mode for achieving zero voltage switching, is used [14]. The output power of a single module is $40 \mathrm{~kW}$, the peak current through the switches is $140 \mathrm{~A}$ and the input

Table I: Isolation transformer efficiency \& loss distribution

\begin{tabular}{ccc}
\hline & Existing transformer & Improved transformer \\
\hline Winding losses & $2330 \mathrm{~W}$ & $1515 \mathrm{~W}$ \\
Core losses & $700 \mathrm{~W}$ & $280 \mathrm{~W}$ \\
Conversion efficiency & $98.8 \%$ & $99.2 \%$ \\
\hline
\end{tabular}



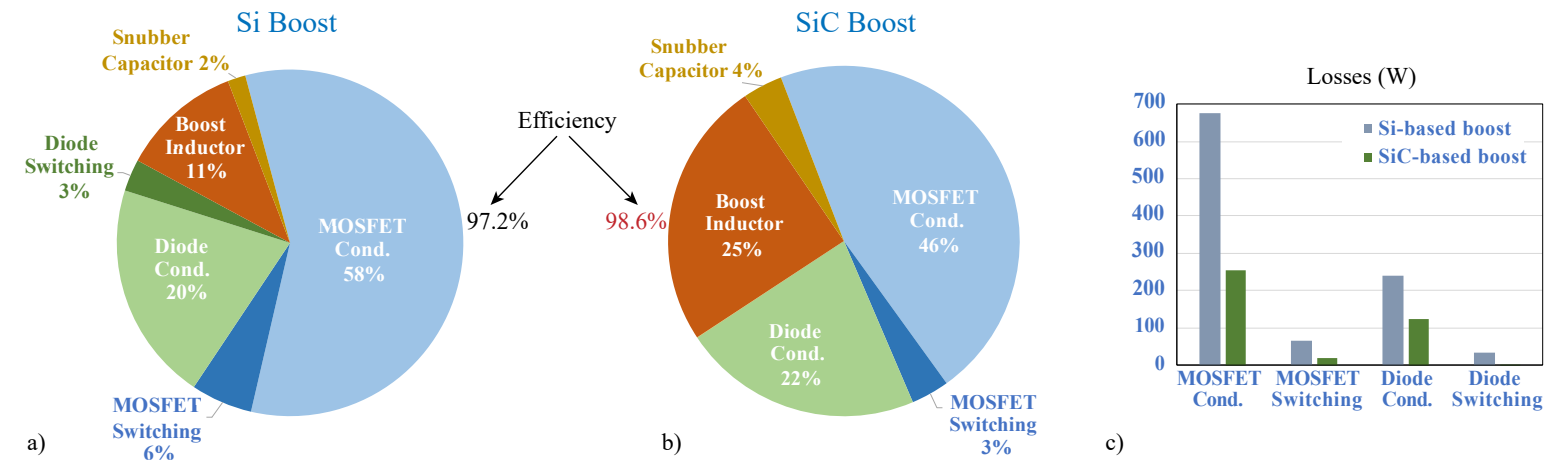

Fig. 2: a) Loss breakdown of the Si-based boost module. b) Loss breakdown of the SiC-based boost module. c) Conduction and switching losses in absolute numbers of each semiconductor for the Si-based and the SiC-based boost module. The efficiency of the Si-based and the SiC-based boost module is $97.2 \%$ and $98.6 \%$ respectively.

voltage is $750 \mathrm{~V}$. In table II, the device arrangement and the model number of the semiconductors for the Si-based and the SiC-based boost module are listed. The conduction and the switching losses of the switches are calculated according to [15] at $70 \mathrm{kHz}$ switching frequency. The boost inductor is designed considering skin, proximity and core losses [8]. The losses of the snubber capacitors are also considered.

The breakdown of the losses and the efficiency of a 40kW boost module are shown in Fig. 2 a) and b) for the Si-based and the SiC-based boost module respectively. In the SiC-based boost, the conduction and the switching losses of the MOSFETs drop by $12 \%$ and $3 \%$ respectively whereas the diode switching losses are almost zero due to the ZVS operation and the ultra-fast $\mathrm{SiC}$ diodes. The Si-based boost reaches a total efficiency of $97.2 \%$ while the SiC-based has an efficiency of $98.6 \%$. The conduction and the switching losses of the semiconductors in absolute numbers are also depicted in Fig. 2 c). In the SiC-based boost module, there is a clear reduction of the conduction and the switching losses compared to the Si-based module.

Table II: Selected boost module devices; ( $x$ syp) for $x$ in series \& $y$ in parallel devices

\begin{tabular}{ccc}
\hline Device & Si-based & SiC-based \\
\hline MOSFET & IPZ65R019C7 (8s2p) & C3M0016120K (4s2p) \\
Diode & APT75DQ120B (4s) & IDW40G120C5B (4s) \\
\hline
\end{tabular}

\section{Droop compensation unit}

The CLIC modulator has four droop compensation units and each unit consists of six interleaved active bouncer modules. The topology of a single bouncer module and the main waveforms are depicted in Fig. 3 a) and b) respectively. Moreover, the bouncer specifications along with the devices that are used for the Si-based and the SiCbased bouncer module are shown in table III. In [16], a detailed explanation of the bouncer operation can be found. During the charging time $t_{\mathrm{ch}}$, the high-side switch $S_{\mathrm{HS}}$, and the short-circuit switch $S_{\mathrm{SC}}$ are on. Consequently, the inductor current slope is known and the losses of the switches can be calculated analytically. Then, the inductor

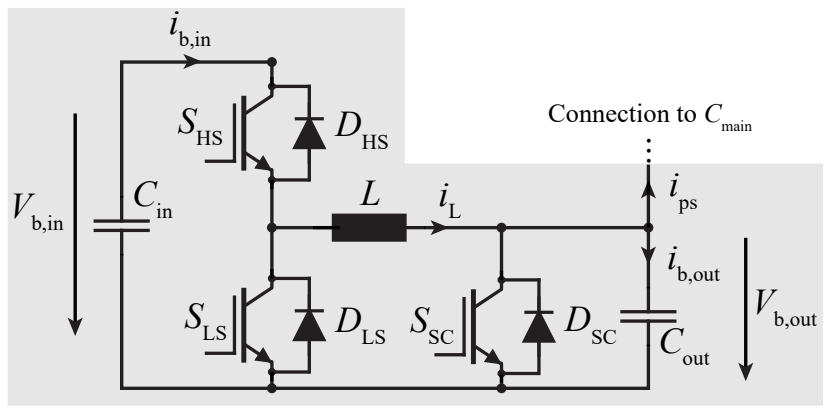

a)

Fig. 3: a) Topology of a bouncer module which consists of a half-bridge $S_{\mathrm{HS}}$ and $S_{\mathrm{LS}}$, an inductor $L$ and a short-circuit switch $S_{\mathrm{SC}}$. b) Bouncer module current and voltage during the pulse operation which is divided in four intervals $t_{\mathrm{ch}}, t_{\mathrm{W}}, t_{\mathrm{flat}}, t_{\mathrm{res}}$ over the repetition period $T_{\text {rep }}$. 
Table III: Selected bouncer module specifications \& devices; $(x p)$ for $x$ in parallel devices

\begin{tabular}{ccc|ccc}
\hline Symbol & Parameter & & Device & Si-based & SiC-based \\
\hline$V_{\mathrm{b}, \text { in }}$ & Input voltage & $450 \mathrm{~V}$ & $S_{\mathrm{HS}}(6 \mathrm{p})$ & IKW50N65F5 & SCTW90N65G2V \\
$V_{\mathrm{b}, \text { out }}$ & Output voltage & $0-300 \mathrm{~V}$ & $D_{\mathrm{HS}}(6 \mathrm{p})$ & IKW50N65F5 & SCTW90N65G2V \\
$I_{\text {nom }}$ & Mean inductor current & $628 \mathrm{~A}$ & $S_{\mathrm{LS}}(2 \mathrm{p})$ & IGW50N65H5 & SCTW90N65G2V \\
$C_{\text {out }}$ & Output capacitance & $66 \mu \mathrm{F}$ & $D_{\mathrm{LS}}(4 \mathrm{p})$ & IDW40E65D1 & IDW40G65C5 \\
$C_{\text {in }}$ & Input capacitance & $2 \mathrm{mF}$ & $S_{\mathrm{SC}}(6 \mathrm{p})$ & IKW50N65F5 & SCTW90N65G2V \\
$L$ & Inductance & $31 \mu \mathrm{H}$ & $D_{\mathrm{SC}}(6 \mathrm{p})$ & IKW50N65F5 & SCTW90N65G2V \\
\hline
\end{tabular}

current slightly decreases during $t_{\mathrm{w}}$ for ensuring equal current sharing between the interleaved branches [16]. During the pulse interval $t_{\text {flat }}$, the converter operates as a typical buck converter with $100 \mathrm{kHz}$ switching frequency and the output voltage of the bouncer rises linearly from 0 to $300 \mathrm{~V}$. An average duty cycle of $33 \%$ is assumed for the calculation of the mean and the RMS current through the switches. The conduction and the switching losses are calculated according to [15] and [17]. For the resonant transition after the pulse interval $t_{\text {res }}$, the components losses are extracted via simulations.

For minimizing the inductor losses, an optimization is performed, which considers skin and proximity losses as well as magnetic core losses [8]. The input and the output capacitor losses are negligible as film capacitors are used.

In Fig. 4 a) and b), the efficiency and the loss distribution are illustrated for the Si-based and the SiC-based bouncer module respectively. It can be observed, that the high side switch contributes $58 \%(51 \%$ switching $+7 \%$ conduction) to the total losses for the Si-based module and it is the main loss component. On the other hand, only $20 \%$ (12\% conduction $+8 \%$ switching) of the total losses come from the high side switch in the SiC-based bouncer. The efficiency of the $\mathrm{Si}$-based and $\mathrm{SiC}$-based module are $91 \%$ and $95.2 \%$ on the bouncer level. However, the power that all the 24 modules $(4 \times 6)$ deliver during the pulse is only $5 \%$ of the total $29 \mathrm{MW}$ pulsed power, and this results in the system level efficiencies of $99.4 \%$ and $99.7 \%$ for the Si-based and the SiC-based bouncer module respectively. In Fig. 4 c), the absolute numbers of the losses for each semiconductor are shown. It is clearly shown that the losses on $S_{\mathrm{HS}}$, which is the main loss contributor, have significantly reduced in the SiC-based bouncer.

\section{Switching unit}

For calculating the losses in the pulse switches, the magnetizing current is neglected. The 5SNA1250B450300 IGBT StakPak switching unit is used in the CLIC modulator. In case SiC MOSFETs are used, the CAB450M12XM3 module is assumed for the calculations. 4 in series and 3 in parallel MOSFETs would be arranged. The conduction and the switching losses are computed based on the data sheet parameters. An efficiency of $98.6 \%$ is achieved for the Si-based switching unit and $99.5 \%$ for the respective SiC-based.

\section{Pulse transformer}

In [18], the transformer winding losses due to skin and proximity effect have been discussed and calculated. A trapezoidal shape is assumed for the pulse current which is analyzed with a Fourier series in order to calculate the skin effect losses. Regarding the proximity effect losses, it is assumed that the windings cover the entire height of the core window and the round conductors of the secondary winding are transformed to a sheet conductor

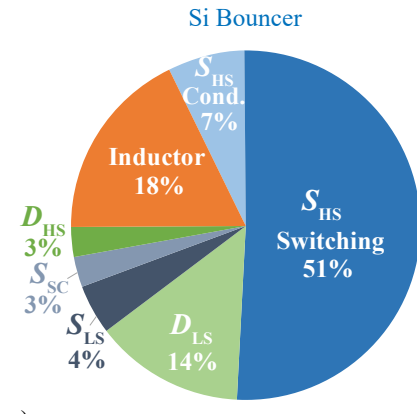

a)

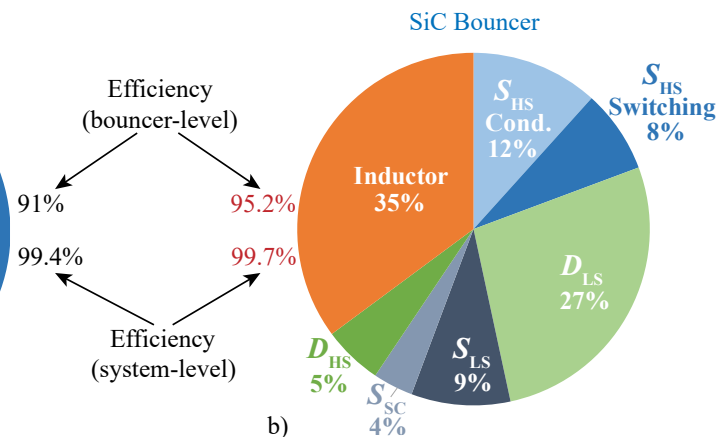

b)

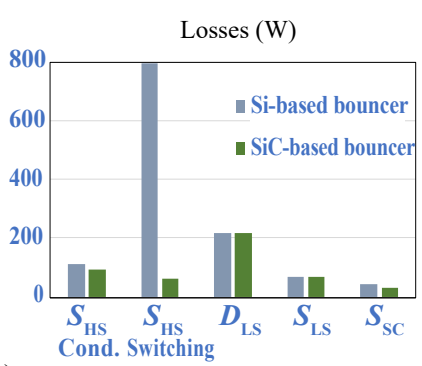

Fig. 4: a) Loss distribution of the Si-based bouncer module. b) Loss distribution of the SiC-based bouncer module. c) Losses in absolute numbers per device for the Si-based and the SiC-based bouncer module. The efficiency of the Si-based and the SiC-based bouncer module is $91 \%$ and $95.2 \%$ on the bouncer level and $99.4 \%$ and $99.7 \%$ on the system level respectively. 
[2]. The core losses are determined by the improved generalized Steinmetz equation by taking into account the pre-magnetization of the core [2].

Another major source of losses in the pulse transformer are the losses due to the pulse shape. To illustrate this, Fig. 5 is provided. Until the flat-top stability (FTS) is reached, energy is lost during the settling time $t_{\text {settle }}$. The same occurs during the fall time $t_{\text {fall }}$ of the pulse. In [19], the pulse efficiency has been defined as the ratio between the energy of an ideal rectangular pulse and the used energy of the real pulse during the flattop period $t_{\text {flat }}$. The real pulse is analyzed in the time domain and, as a result, it is possible to integrate during the settling and fall time to obtain the energy loss.

The conversion efficiency of the split two-core pulse transformer is obtained through an optimization procedure. A detailed explanation of the optimization algorithm steps can be found in [4], but an overview of the algorithm is illustrated in Fig. 6 and it is explained briefly here. The main optimizer sets the lower and upper limits of the optimization variables and initiates the genetic algorithm. In the parameters definition section, the user sets the electrical characteristics of the modulator, such as the desired pulsed power and voltage, the pulse requirements, such as the FTS criteria and maximum permissible overshoot, and other predefined geometrical characteristics. Based on these parameters, the volume of the transformer and the tank is obtained through the transformer geometry formulation sub-module. Then, the maximum electric field $E_{\max }$ is calculated on the surface of the high voltage conductors by using the charge simulation method [8]. If $E_{\max }$ is less than the preset threshold $E_{\mathrm{th}}$, the procedure continues with the analytical calculation of the leakage inductance $L_{\sigma}$ and distributed capacitance $C_{\mathrm{d}}$ of the transformer geometry [1]. Finally, the pulse shape is analyzed in the time domain by using the secondary-referred electrical equivalent circuit of the transformer and, if the pulse requirements are met, the

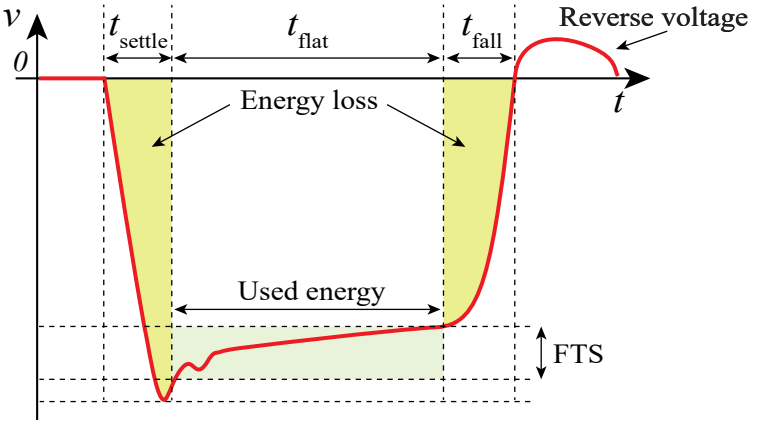

Fig. 5: Pulse shape of CLIC modulator. Once the pulse lies into flat-top stability (FTS) band, the flat-top interval $t_{\text {flat }}$ begins.

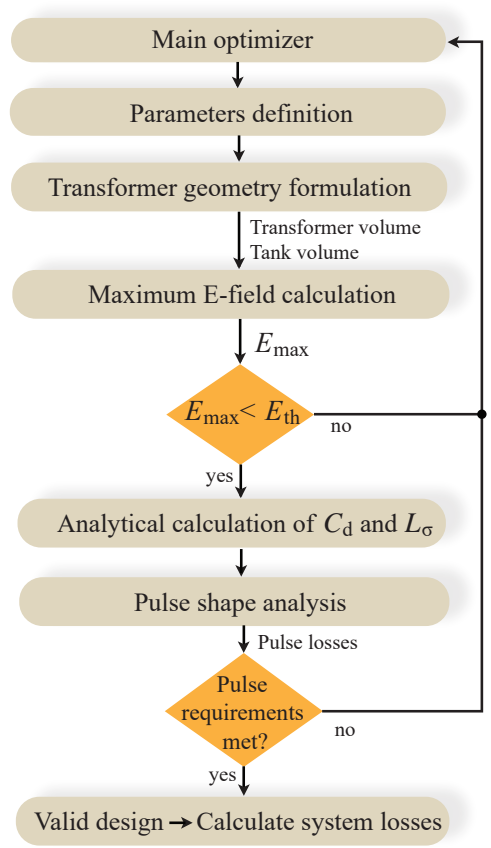

Fig. 6: Flowchart of the pulse transformer optimization. design is considered valid and the system losses are calculated. The system losses include the winding and the core losses of the transformer as well as the losses due to the pulse shape.

In addition, the length of the high voltage cable, which connects the transformer to the klystron, and the type of the insulating oil affect the transformer efficiency, as they change the total distributed capacitance of the transformer and therefore, the intervals $t_{\text {settle }}$ and $t_{\text {fall }}$ also change. To visualize this dependency, Fig. 7 is provided. It is observed that the use of mineral oil instead of natural ester oil, can result in higher transformer efficiencies at the same cable length $l_{\mathrm{HV}}$. It is also shown that the higher the cable length, the lower the conversion efficiency of the transformer due to the additional paracitic capacitance of the cable. The selected pareto point for the CLIC system results in a transformer conversion efficiency of $96.7 \%$ including winding, core, and pulse shape losses. Approximately $4 \%$ are winding losses, $8 \%$ core losses, and $88 \%$ are losses due to the pulse shape. However, in [4], it has been shown that a $97.5 \%$ efficiency could be achieved if a three-core arrangement, mineral oil, and a SiFE with $25 \mu \mathrm{m}$ lamination core material would be chosen for the CLIC system instead of two-core and natural ester type of oil. However, this solution would lead to a transformer with a significantly higher volume.

\section{CLIC Efficiency Results}

Combining the results of the previous section, the total efficiency of the existing modulator and the improved $\mathrm{SiC}$-based can be obtained. Using $\mathrm{SiC}$ semiconductors along with the other suggested improvements can lead to a substantially higher efficiency for the CLIC modulator system. In Fig. 8 a) and b), the breakdown of the losses for each sub-system of the existing Si-based and the improved SiC-based modulator are shown respectively. The 


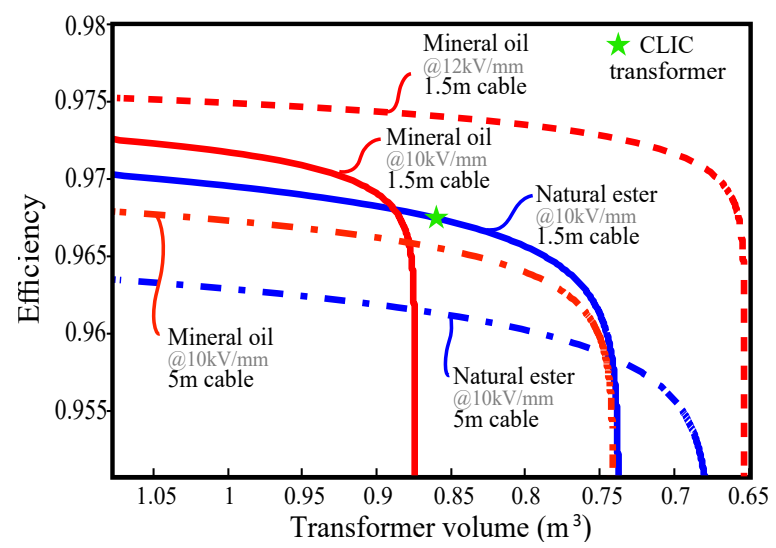

Fig. 7: Pareto limit between conversion efficiency and transformer volume with 1) ester oil, $l_{\mathrm{HV}}=5 \mathrm{~m}$ with $E_{\max }=10 \mathrm{kV} /$ $\mathrm{mm}, 2$ ) ester oil, $l_{\mathrm{HV}}=1.5 \mathrm{~m}$ with $\left.E_{\max }=10 \mathrm{kV} / \mathrm{mm}, 3\right)$ mineral oil, $l_{\mathrm{HV}}=5 \mathrm{~m}$ with $\left.E_{\max }=10 \mathrm{kV} / \mathrm{mm}, 4\right)$ mineral oil, $l_{\mathrm{HV}}$ $=1.5 \mathrm{~m}$ with $\left.E_{\max }=10 \mathrm{kV} / \mathrm{mm}, 5\right)$ mineral oil, $l_{\mathrm{HV}}=1.5 \mathrm{~m}$ with $E_{\max }=12 \mathrm{kV} / \mathrm{mm} \mathrm{[2]}$.

improved system shows an efficiency of $93.6 \%$ and the existing $88.7 \%$, which means a $4.9 \%$ efficiency increase. In the improved system, the pulse transformer losses dominate (39\%), which is $11 \%$ larger than the existing modulator. In both systems, the pulse transformer constitutes the main loss contributor. In the improved modulator, the PFC, the switching unit and the boost converter have a lower contribution to the total losses compared to the existing modulator system. Moreover, the obtained efficiencies for each conversion stage are shown in the table of Fig. 8 c) while in Fig. 8 d), the total power losses for the two systems are depicted.

\section{ESS Modulator Topology}

The ESS modulator topology along with the main specifications are presented in [20] and a schematic overview of the system is given in Fig. 9. A PFC charging system is connected to the input $400 \mathrm{~V}$ AC mains which forms an $800 \mathrm{~V}$ DC-link bus. Next, 2 series/parallel resonant converter basic modules (SPRC-Bm) with a $400 \mathrm{~V}$ DClink voltage are connected in series to the $800 \mathrm{~V}$ DC-link bus. A single SPRC-Bm is a Si MOSFET-based full bridge, followed by a resonant tank, a medium frequency transformer and an output rectifier. At the output, the 2 SPRC-Bms are connected in parallel creating an input series, output parallel (ISOP) stack. In order to reach the desired output voltage and power levels, 9 ISOP stacks are connected in parallel at the input and in series at the output. A balancing circuit is also connected to the input of each ISOP stack ensuring an equal voltage sharing across the capacitors $C_{\mathrm{DLi}}$ after each pulse. A filter capacitor $C_{\mathrm{f}}$ and the klyston load is attached to the output of the modulator. The converter operates between $100-110 \mathrm{kHz}$ for compensating the droop of the pulse. For the calculations an average switching frequency of $105 \mathrm{kHz}$ is assumed.

\section{Efficiency of ESS Stages}

In this section, the efficiency of each individual stage of the ESS solid-state pulse modulator is presented for the Si-based and for the improved SiC-based system. The efficiency calculation for the improved SiC-based system are based on the configuration that is illustrated in Fig. 10. In the improved SiC-based modulator, the balancing circuit is not required and the input voltage level for each SPRC-Bm is $800 \mathrm{~V}$.
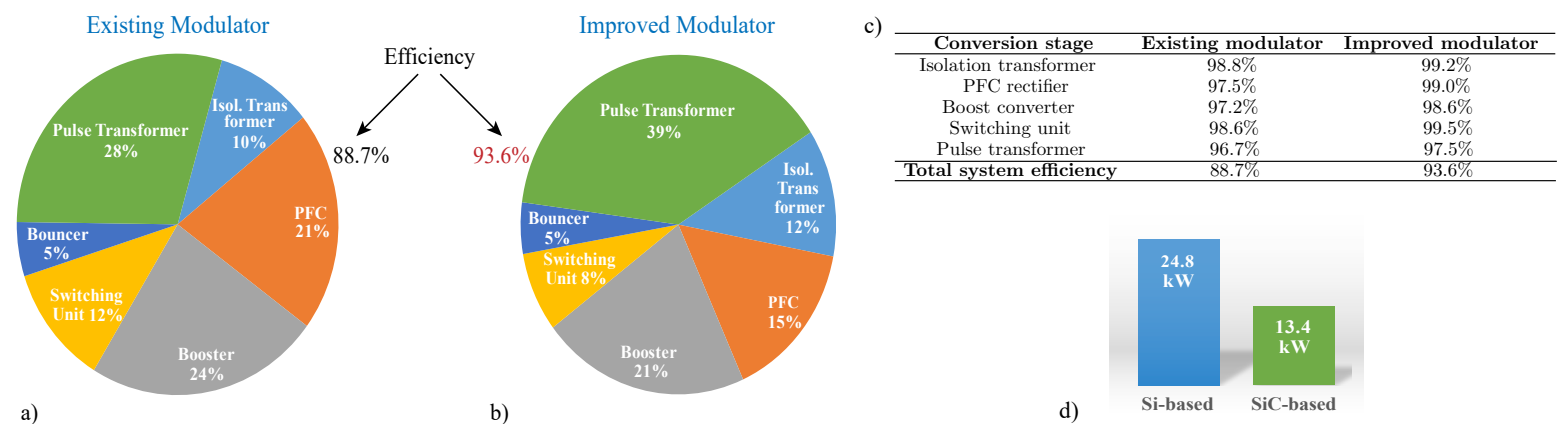

Fig. 8: a) Loss distribution of the existing Si-based CLIC modulator. b) Loss distribution of the improved SiC-based CLIC modulator. c) Conversion stages \& total system efficiency results for the CLIC modulator. d) Total losses of the Si-based \& the SiC-based CLIC modulator. The existing modulator shows an $88.7 \%$ efficiency and the improved modulator has $93.6 \%$. 


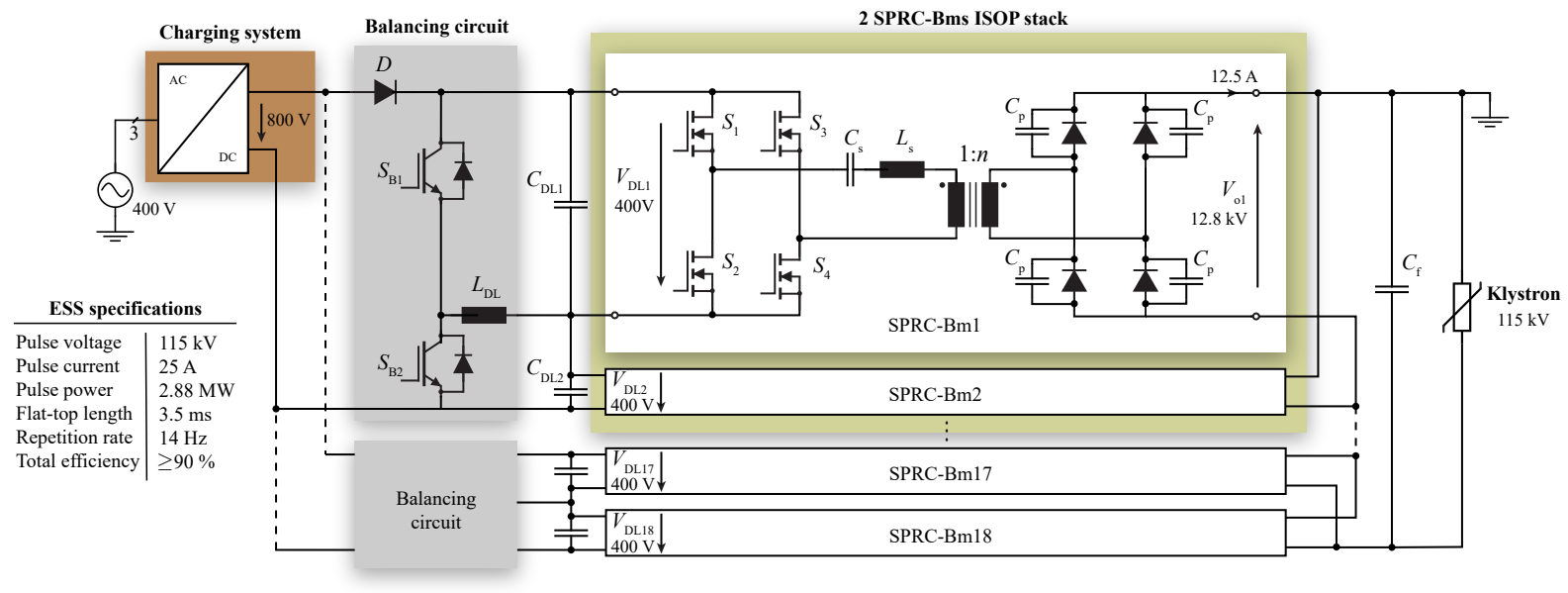

Fig. 9: ESS pulse modulator schematic overview consisting of: Charging system, balancing circuits, two series/parallel resonant converter basic modules (2 SPRC-Bms) in input series output parallel (ISOP) stacks, and klystron load.

\section{Charging system}

The input charging system is a standard industry PFC boost converter unit from REVCON with an efficiency of 98\% according to the data sheet [21]. A SiC-based solution for the PFC converter could, potentially, have a $99 \%$ efficiency as discussed in section 3.

\section{Series/Parallel Resonant Converter-Basic Module}

The losses of the isolated DC-DC converter occur on the semiconductors, the passive components and the transformer. The analysis of the efficiency calculation starts with the losses in the power switches. Output filter capacitor losses are not considered as film capacitors are used with very low ESR.

- Semiconductors losses: In the first step, the losses of the semiconductors are derived. The selected device arrangement per each branch of the low voltage (LV) and the high voltage (HV) side of the converter is listed in Table IV for the Si-based and the SiC-based module. For the conduction loss calculation, the average and the RMS values of the currents through all the switching components are calculated analytically with the formulas presented in [9]. Then, the conduction losses are computed as proposed in [15]. The MOSFETs and the diodes turn-on under zero voltage and therefore, only turn-off losses occur which are extracted via simulations [9]. Finally, the switching losses of the anti-parallel diodes and the rectifier diodes are considered to be negligible because they turn-off under low di/dt and ultra-fast recovery diodes are used for the rectifier stage.

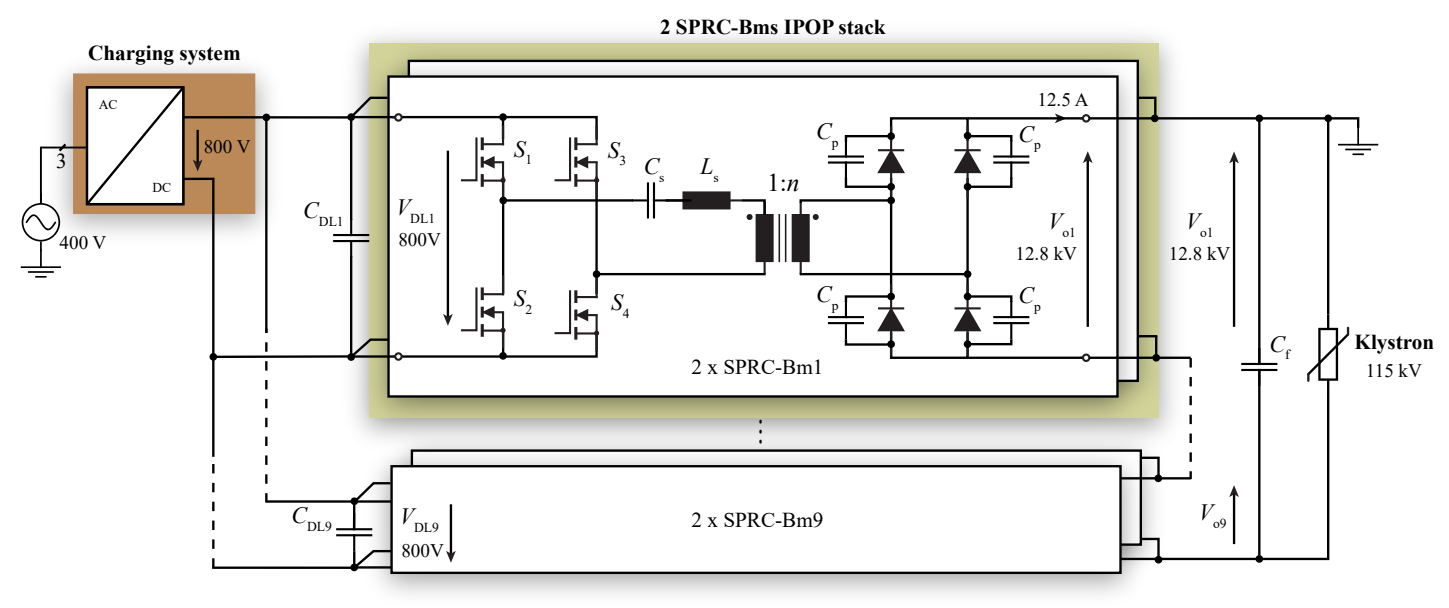

Fig. 10: Improved SiC-based ESS pulse modulator schematic overview consisting of: Charging system, two series/parallel resonant converter basic modules (2 SPRC-Bms) in input parallel output parallel (IPOP) stacks, and klystron load. Comparing to the existing ESS modulator, the balancing circuit is not required and the input voltage level for each SPRC-Bm is $800 \mathrm{~V}$ in the improved modulator. The output power and voltage levels of the SiC-based system remain the same. 
Table IV: Selected devices for the SPRC-Bm; ( $x$ syp) for $x$ in series \& $y$ in parallel devices in each branch.

\begin{tabular}{ccc}
\hline Device & Si-based & SiC-based \\
\hline MOSFET (LV) & STY139N65M5 (6p) & IMZ120R030M1H (6p) \\
Diode (HV) & APT60DQ120SG (36s) & C5D25170H (26s2p) \\
\hline
\end{tabular}

- Passive components losses: At this point, the loss calculation of the series capacitors $C_{\mathrm{s}}$, the parallel capacitors $C_{\mathrm{p}}$ as well as the series inductance $L_{\mathrm{s}}$ is described. The series capacitor consists of 896 capacitors of 15 $\mathrm{nF}$ connected in a series/parallel arrangement. The parallel capacitor of the rectifier has 864 capacitors (216 per diode branch) of $100 \mathrm{nF}$ in a series/parallel connection. The losses for the parallel capacitance are given by the following relation [9]

$$
P_{C_{\mathrm{p}}}=D \cdot 2 \pi f_{\mathrm{s}} \cdot C_{\mathrm{p}} \cdot \frac{V_{C_{\mathrm{p}, 1}}^{2}}{2} \cdot \tan \delta_{C_{\mathrm{p}}}
$$

where $D$ is the duty cycle of the pulse, $f_{\mathrm{s}}$ is the average switching frequency, $V_{C_{\mathrm{p}, 1}}$ the fundamental component of the voltage across $C_{\mathrm{p}}$ and $\tan \delta_{C_{\mathrm{p}}}$ is the loss factor. The loss factor of the NP0 ceramic material of the capacitor is given in the data sheet. The same formula is applied for the series capacitor.

The required series inductance is an air toroid which offers the advantage of no core losses, no saturation as well as low stray magnetic field [22]. The toroid is made of litz wires and the winding losses calculation is explained in [22]. Given that in the improved SiC-based modulator the input voltage level is twice the voltage level of the existing system and the power level remains the same, this results to half of the current which flows through $L_{\mathrm{S}}$. Assuming that the DC resistance of $L_{\mathrm{S}}$ can take a value that it is twice the current one, this leads to half of the power losses for the $L_{\mathrm{S}}$ in the improved SiC-based modulator.

- Transformer losses: For the primary and the secondary winding of the transformer, litz wire is used and ferrite K2008 as core material. Due to the almost sinusoidal flux density, the standard Steinmetz equation is used for the core losses calculation [23]. However, according to the data sheet, a $20 \%$ core losses reduction is feasible in case N97 core material is used [24].

The total winding losses $P_{\mathrm{wdg}}$ of the transformer are calculated with the same way as the winding losses of the toroid, except slight differences in the external magnetic field intensity analytic formulas [9], and they are given by the relation

$$
P_{\mathrm{wdg}}=D \cdot N_{\mathrm{s}} \cdot l_{\mathrm{m}}\left(\frac{N F_{\mathrm{s}} \hat{I}^{2}}{N_{\mathrm{s}}^{2}}+\sum_{n=1}^{N} G_{\mathrm{s}} H_{\mathrm{avg}, n}\right)
$$

with

$$
\hat{H}_{\mathrm{avg}, n}=\frac{1}{\pi r_{\mathrm{a}}^{2}} \int|H|^{2} d A_{\mathrm{n}},
$$

where $N_{\mathrm{s}}$ is the number of strands per bundle, $l_{\mathrm{m}}$ is the mean turn length, $N$ is the number of turns, $F_{\mathrm{S}}$ and $G_{\mathrm{S}}$ are the skin and proximity effect factors respectively, and $\hat{I}$ stands for the peak current of the winding. For the average magnetic field intensity of each turn $H_{\mathrm{avg}, n}$, the outer radius of the litz wire bundle $r_{\mathrm{a}}$ as well as the norm of the magnetic field density of each bundle $|H|$ over each turn cross section $A_{n}$ are needed. In [23], a detailed analysis is presented for calculating the magnitude of the field intensity $|H|$.

In Fig. 11 a) and b), the loss distribution of the Si-based and the SiC-based SPRC-Bm is depicted respectively, excluding the pulse shape losses at this point. The improved SiC-based module has an efficiency of $94.4 \%$ which is $1.5 \%$ higher than the existing Si-based SPRC-Bm presents. In the improved module, the main power loss factors are the full bridge and the transformer with $40 \%$ and $18 \%$ respectively, while the contribution of the series inductor dropped from $21.9 \%$ to $14 \%$. In addition, the losses in absolute numbers for each component of the Si-based and the SiC-based SPRC-Bm are presented in Fig. $11 \mathrm{c}$ ), showing the reduction of the losses in case of a SiC-based SPRC-Bm.

In order to also include the pulse shape losses to the overall system efficiency calculation, Fig. 12 is provided [20]. In Fig. 12 a), the measured output voltage of the built modulator system is shown where $V_{\text {out }}$ (blue line) stands for the actual output voltage and $V_{\text {out,avg }}$ (green line) is the averaged pulse voltage which is used for computing the rise time $t_{\text {rise }}$ and the fall time $t_{\text {fall }}$. Fig. $\left.12 \mathrm{~b}\right)$ illustrates a zoomed version during the rise time ( 0 to $\left.0.99 V_{\text {nom }}\right)$ of 

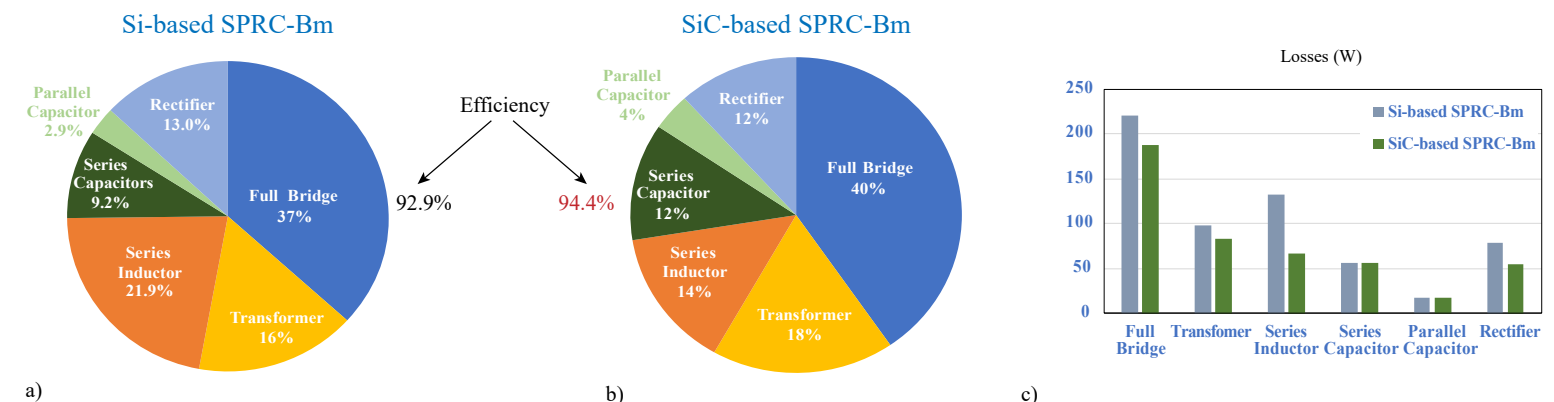

Fig. 11: a) Loss distribution of the Si-based SPRC-Bm. b) Loss distribution of the SiC-based SPRC-Bm. c) Losses in absolute numbers per component for the Si-based and the SiC-based SPRC-Bm. The efficiency of the Si-based and the SiC-based SPRC$\mathrm{Bm}$ is $92.9 \%$ and $94.4 \%$ respectively. The pulse shape losses are not included in the resonant converter efficiency calculation.

the pulse whereas Fig. $12 \mathrm{c}$ ) presents the zoomed version of the pulse during the fall time ( $V_{\text {nom }}$ to $\left.0.1 V_{\text {nom }}\right)$. The quantity $V_{\text {nom }}$ stands for the nominal output voltage, namely $115 \mathrm{kV}$. The areas $K_{1}$ and $K_{2}$ correspond to the lost energy during the rise time and the fall time respectively. The pulse efficiency $\eta_{\text {pulse }}$ is the ratio among the ideal rectangular pulse and the real pulse and it is given by the following relation

$$
\eta_{\text {pulse }}=\left(\frac{K_{\text {ideal }}}{K_{\text {real }}}\right) \cdot 100 \%
$$

where

$$
K_{\text {ideal }}=V_{\text {nom }} \cdot\left(t_{1}-t_{\text {rise }}\right), \quad K_{\text {real }}=\int_{0}^{t_{2}} V_{\text {out }}(t) \mathrm{d} t
$$

\section{ESS Efficiency Results}

The analysis from section 6, results in the efficiency values for each conversion stage of the existing Si-based and the improved SiC-based ESS modulator which are listed in Table V. More specific, the improved modulator has an electrical system (PFC + Resonant converter) efficiency of $93.5 \%$ which is $2.9 \%$ higher than the existing system. Assuming equal pulse losses for both systems, the improved modulator shows an overall efficiency of $91.4 \%$ while the existing modulator has $88.6 \%$.

To illustrate the loss distribution for both systems, Fig. 13 is given. In the improved modulator, the main loss contributor are the pulse shape losses with $25.4 \%$ whereas, in the existing system, the losses of the full-bridge dominate with $23.7 \%$. In the improved modulator, the losses in the resonant tank drop from $20.1 \%$ to $17.8 \%$ compared to the existing modulator, which is a $2.3 \%$ decrease. The PFC unit contributes $11.4 \%$ to the overall efficiency of the SiC-based system while in the existing system, $16.8 \%$ of the losses are due to the PFC charging unit. On the contrary, the loss share of the rectifier and the transformer, rise from $10.3 \%$ to $14 \%$ and from $10.5 \%$ to $12.1 \%$, respectively, in the improved modulator system.
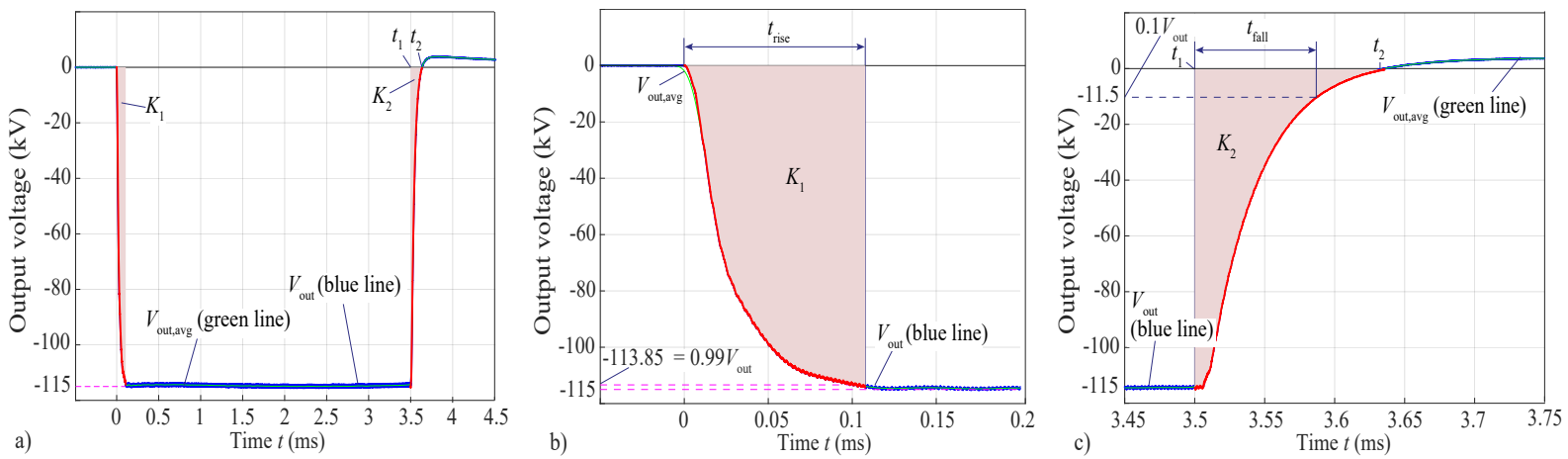

Fig. 12: a) Measured output voltage $V_{\text {out }}$ (blue line) and averaged output voltage (green line). b) Zoomed view of the output voltage during the rise time $t_{\text {rise }}$. c) Zoomed view of the output voltage during the fall time $t_{\text {fall }}$. The areas $K_{1}$ and $K_{2}$ correspond to the lost transferred energy that the klystron cannot use during the rise time and the fall time respectively [20]. 
Table V: Conversion stages \& total system efficiency results for the existing and the improved ESS modulator.

\begin{tabular}{ccc}
\hline & Existing modulator & Improved modulator \\
\hline PFC rectifier & $98.0 \%$ & $99.0 \%$ \\
Resonant converter & $92.9 \%$ & $94.4 \%$ \\
Pulse shape & $97.8 \%$ & $97.8 \%$ \\
\hline Electrical system efficiency & $90.6 \%$ & $93.5 \%$ \\
Total system efficiency & $88.6 \%$ & $91.4 \%$ \\
\hline
\end{tabular}

Existing Modulator

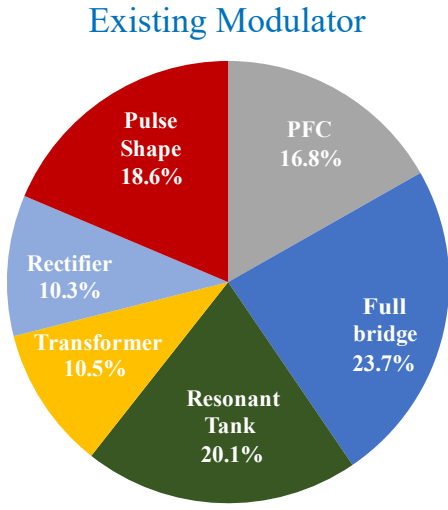

$88.6 \%$

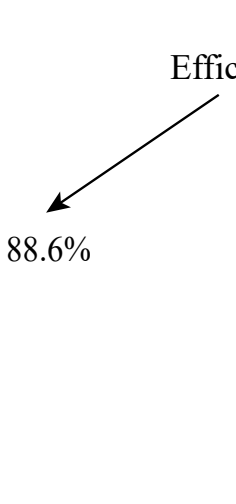

Efficiency
Improved modulator

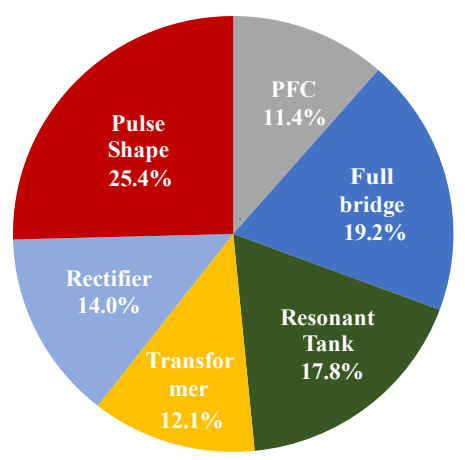

b)

Fig. 13: a) Loss distribution of the existing Si-based ESS modulator. b) Loss distribution of the improved SiC-based ESS modulator. The existing system reaches an efficiency of $88.6 \%$ and the improved system has an efficiency of $91.4 \%$ including pulse shape losses.

\section{Conclusion}

In this paper, the loss distribution of all conversion stages of the CLIC and the ESS pulse modulator systems is presented. The analysis leads to the efficiency calculation of each stage and, finally, the total efficiency for the CLIC modulator is obtained as $88.7 \%$ while the ESS modulator shows an of efficiency $88.6 \%$, including pulse shape losses for both systems. Furthermore, it is investigated how much $\mathrm{SiC}$ devices could improve the efficiency of the existing modulators which are currently based on Si semiconductors. The results indicate that the installation of $\mathrm{SiC}$ semiconductors, along with the additional proposed improvements, can lead to an increased overall efficiency which is calculated to be $93.6 \%$ for the CLIC system and $91.4 \%$ for the ESS system. Essential parameters of the CLIC modulator, which affect the system efficiency and should be considered during the design process, are the type of the insulating oil of the transformer, the length of the high voltage cable, which connects the transformer to the klystron load, as well as the core material and the lamination of the transformer. Finally, further improvements are possible for the performance of the SiC-based modulators in case a new overall optimization would be carried out for both systems. 


\section{References}

[1] M. Jaritz and J. Biela, "Optimal design of a modular series parallel resonant converter for a solid state 2.88 MW/115-kV long pulse modulator," IEEE Trans. on Plasma Science, vol. 42, no. 10, pp. 3014-3022, Oct. 2014.

[2] S. Blume, M. Jaritz, and J. Biela, "Design and optimization procedure for high-voltage pulse power transformers," IEEE Trans. on Plasma Science, vol. 43, no. 10, pp. 3385-3391, Oct. 2015.

[3] W. Crewson, M. Lindholm, and K. Elmquist, "Green pulsed power achieved by efficient solid state pulsed power technology," in IEEE Pulsed Power Conf., June 2011, pp. 1471-1473.

[4] S. Blume and J. Biela, "Optimal transformer design for ultraprecise solid state modulators," IEEE Trans. on Plasma Science, vol. 41, no. 10, pp. 2691-2700, Oct. 2013.

[5] D. Gerber and J. Biela, "Design of an ultraprecise 127-MW/3- $\mu$ s solid-state modulator with split-core transformer," IEEE Trans. on Plasma Science, vol. 44, no. 5, pp. 829-838, May 2016.

[6] W. Hartmann, R. Fleck, R. Graba, and M. Hergt, "Characterization of commercial IGBT modules for pulsed power applications," in 19th IEEE Pulsed Power Conf. (PPC), June 2013.

[7] L. M. Redondo, A. Kandratsyeu, and M. J. Barnes, "Marx generator prototype for kicker magnets based on SiC MOSFETs," IEEE Trans. on Plasma Science, vol. 46, no. 10, pp. 3334-3339, Oct. 2018.

[8] M. S. Blume, "Highly efficient pulse modulator system with active droop compensation for linear colliders," Ph.D. dissertation, ETH Zürich, 2016.

[9] M. Jaritz, "Solid-state modulator for generating high voltage pulses in the ms-range with high output power," Ph.D. dissertation, ETH Zürich, 2018.

[10] ABB, "Amorphous core distribution transformers," April 2015.

[11] B\&R Industrial Automation GmbH, “ACOPOS Multi User’s Manual,” April 2018.

[12] A. Stupar, T. Friedli, J. Miniböck, M. Schweizer, and J. W. Kolar, "Towards a 99\% efficient three-phase buck-type PFC rectifier for 400 V DC distribution systems,” vol. 27, no. 4, April 2012, pp. 1732-1744.

[13] J. Biela, J. W. Kolar, and G. Deboy, "Optimal design of a compact 99.3\% efficient single-phase PFC rectifier," in IEEE Applied Power Electronics Conf. and Exposition (APEC), Feb. 2010, pp. 1397-1404.

[14] S. Blume, D. Gerber, and J. Biela, "High precision, low ripple 3kV capacitor charger," in IEEE Int. Power Modulator and High Voltage Conf. (IPMHVC), July 2016, pp. 113-119.

[15] D. Graovac, M. Pürschel, and A. Kiep, "MOSFET power losses calculation using the data-sheet parameters," July 2006, Application Note, V 1. 1.

[16] S. Blume and J. Biela, "Design procedure of an active bouncer for an ultra precise long pulse solid state modulator," in IEEE Pulsed Power Conf., June 2013.

[17] D. Graovac and M. Pürschel, "IGBT power losses calculation using the data-sheet parameters," Jan. 2009, Application Note, V 1. 1.

[18] J. Biela, "Optimierung des electromagnetisch integrierten serien-parallel resonanzkonverters mit eingeprägtem ausgangsstrom,” Ph.D. dissertation, ETH Zürich, 2005.

[19] D. Aguglia and E. Sklavounou, "Klystron modulators capacitor chargers design compromises for ac power quality increase of the compact linear collider (CLIC)," in Int. Symposium on Power Electronics, Electrical Drives, Automation and Motion (PCIM), June 2012, pp. 1535-1541.

[20] M. Jaritz and J. Biela, "System design and measurements of a 115-kV/3.5-ms solid-state long-pulse modulator for the european spallation source," IEEE Trans. on Plasma Science, vol. 46, no. 10, pp. 3232-3239, Oct. 2018.

[21] REVCON, "Power feed and feedback module REVCON DC(S)," May 2019. 
[22] M. Jaritz, S. Blume, D. Leuenberger, and J. Biela, "Experimental validation of a series parallel resonant converter model for a solid state 115-kV long pulse modulator," IEEE Trans. on Plasma Science, vol. 43, no. 10, pp. 3392-3398, 2015.

[23] M. Jaritz, S. Blume, and J. Biela, "Design procedure of a $14.4 \mathrm{kV}, 100 \mathrm{kHz}$ transformer with a high isolation voltage (115 kV)," IEEE Trans. on Dielectrics and Electrical Insulation, vol. 24, no. 4, pp. 2094-2104, 2017.

[24] TDK, "Ferrites and accessories, SIFERRIT material N97," May 2017. 